\title{
Improving systems through the use of improvement and safety science: A case study in pain pump and patient transfer processes
}

\author{
Shea Polancich ${ }^{* 1}$, Terri Poe ${ }^{1,2}$, Linda Moneyham ${ }^{1}$ \\ ${ }^{1}$ School of Nursing, University of Alabama at Birmingham, Birmingham, United States \\ ${ }^{2}$ University of Alabama at Birmingham Hospital, Birmingham, United States
}

Received: January 26, 2015

Accepted: April 15, 2015

Online Published: April 27, 2015

DOI: $10.5430 /$ jnep.v5n7p45

URL: http://dx.doi.org/10.5430/jnep.v5n7p45

\begin{abstract}
Introduction: Using a pain pump or patient controlled analgesia (PCA) has been shown to be an effective method of pain control. The goal for the bedside nurse is to maintain patient safety while allowing patient autonomy for pain control. Healthcare organizations define established processes, protocols and standards that are relevant to the safe performance of actionable tasks. This case study describes an opportunity for improvement in process variation associated with the use of pain pumps/PCAs and patient transport.

Methods: In this case study, a perceived process variation was identified in the transport of hospitalized inpatients to different locations with pain pumps. A systematic approach to mitigating process variation was implemented using an inter-professional team approach including the use of a team charter, defined methodological framework incorporating the Model for Improvement, Plan-Do-Study-Act cycle, microsystem analysis, indirect event review, and evidence appraisal.

Results: Process variation in the management of patients with a pain pump being transported throughout the healthcare organization was the result of system gaps in communicating and managing organizational policies and procedures. The results of the analysis of the pain pump transfer project highlighted the following: system-based improvement, leadership accountability, and education-practice partnerships.

Conclusions: Managing the transport of patients with a pain pump within a healthcare organization is a process that should be standardized, communicated and well understood by all providers. From a broader perspective, this case study highlighted an organizational need to develop a standardized process for learning from process variation, but also for developing tools and competencies within the organization for this work.
\end{abstract}

Key Words: Pain pump, System-based improvement, Patient safety

\section{INTRODUCTION}

Patients who are experiencing pain following surgery, trauma, or acute exacerbations associated with chronic conditions often utilize pain control methods such as a pump, or patient controlled analgesia (PCA) in order to effectively man- age pain. The most common medication delivered via a PCA pump are opioids in which the patient is able to selfadminister prescribed doses of medication through a controlled mechanism (a safety mechanism that limits overdose) over time to remain pain-free. ${ }^{[1]}$ The use of a pain pump

\footnotetext{
*Correspondence: Shea Polancich; Email: polancs@uab.edu; Address: NB 352, School of Nursing, 1720 2nd Ave S., University of Alabama at Birmingham, Alabama, United States.
} 
or PCA has been shown to be an effective method of pain control for the patient given appropriate patient selection criteria and rigorous attention to safe administration of medication that most specifically fits the patient situation. For the practicing bedside nurse, the goal is to maintain patient safety while allowing patient autonomy for pain control. ${ }^{[2]}$ In addition, it is the role of nurse to ensure that protocols, processes, and procedures for the use of the pain pump within an organization are maintained and followed. ${ }^{[3]}$

Pain management for the hospitalized inpatient is an important factor in promoting patient healing, and also impacts patient satisfaction during their hospital stay. PCA pain pump management has been found, historically, to be an effective method for controlling patient pain and a positive patient satisfier. ${ }^{[4]}$ Two factors identified in the research literature as important to nursing care delivery are: 1) ensuring that the processes for using PCA emphasize safe narcotic administration; and 2) assessment of the patient through the duration of PCA therapy.

Within any organization, there are established processes, protocols and standards that are relevant to the safe performance of actionable tasks for all levels of providers. Nursing is no exception, and typically, nursing actions are underpinned by evidenced based guidelines for tasks found in documents such as Lippincott standards, ${ }^{[5]}$ as well as in organizational specific protocols and processes. These standards, processes, or protocols provide direction for care, and limit variation in practice when appropriate adherence is observed.

The following case study describes an opportunity for improvement within an academic organization where variation in processes, standards, and protocols associated with the use of pain pumps/PCAs were examined in order to revise and standardize processes for effective and safe pain management.

\section{The pain pump and patient transfer process-variation in practice and processes}

A group of nurse leaders at a University Medical Center were asked by the Chief Nursing Officer (CNO) to review the process for intra-hospital transfers of patients with pain pumps. This request was triggered by a verbal report to the CNO that there was variation across units in the process of transferring patients with a pain pump; some patients were being transferred without pumps and consequently, were experiencing periods of inadequate pain management. It was expected that such periods of inadequate pain management were associated with lower patient satisfaction scores for the Hospital Consumer Assessment of Healthcare Providers and Systems (HCAHPS) pain management domain. ${ }^{[6]}$ A small task force was chartered to understand current practices in transferring patients with pain pumps across the medical center.

The CNO mobilized the strong academic/clinical partnership with the School of Nursing with the aim of engaging Doctor of Nursing Practice (DNP) faculty and students to lead and facilitate the quality improvement process with identified clinical teams in order to test a structured method of chartering and improving systems and processes using improvement and safety science methods. A nurse improvement scientist with a joint appointment in the School of Nursing and the University Medical Center was tapped to lead the collaboration, mentor facilitators, and serve as an advisor to the quality improvement project team. The faculty-improvement scientist used improvement and safety science methods to develop a standardized charter that would analyze the patient transfer process with the pain pump using a microsystem analysis, ${ }^{[7]}$ the model for improvement and Plan-Do-Study-Act (PDSA) small test of change cycle process, ${ }^{[8]}$ and indirect event analysis for understanding the "whys" of process variation

\section{METHODS}

In this case study, a perceived gap or process variation was identified in the transport of hospitalized inpatients to different locations with pain pumps. This process variation was examined using the microsystem analysis and the model for improvement and PDSA process. According to Sherwood and Barnsteiner, ${ }^{[9]}$ improvement is one of the key components of creating a culture of excellence in nursing practice. As such, nursing and care delivery processes should be continually improved using the following four components: assess, plan/prioritize, implement/execute, and evaluate. ${ }^{[9]}$ This improvement process used to understand the gap/process variation will be detailed in the sections to follow.

The Model for Improvement (MFI) ${ }^{[10]}$ was specifically developed to guide the improvement process using three guiding questions that are outlined in Figure 1: 1) What am I trying to accomplish? 2) How will I know a change is an improvement? and 3) What changes can I implement that will result in an improvement? These three questions guide the development of an improvement intervention that is tested using a small test of change process or a rapid cycle improvement process, called a PDSA cycle ${ }^{[8]}$ as shown in Figure 2 . The details of the use of the improvement process are described through the case study, where the use of the model is further delineated.

In addition, integral to any improvement is a clear understanding and quantification of a gap or area for improvement. The Microsystem Analysis process, developed out 
of Dartmouth, ${ }^{[11]}$ is shown in Figure 3. It was used to provide structure for quantifying the opportunity for improvement. The "5 P's" of the microsystem analysis, namely, patients/populations, processes, patterns, providers, and purpose, were analyzed prior to developing or implementing an intervention. The analysis was completed with the "Plan" phase of the improvement PDSA to provide a foundation and a baseline for any change.

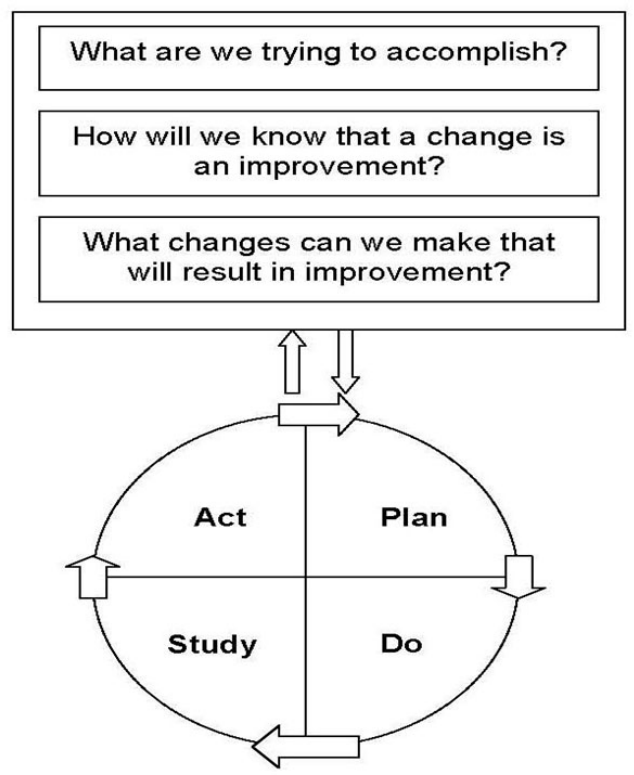

Figure 1. Model for improvement

For the purposes of this improvement project, the microsystem was defined specifically as nursing services and processes surrounding patient transport with a pain pump. Dur- ing the hospital stay, patients who have a need for more effective pain management may be placed on a pain pump (or patient controlled analgesia). With a "purpose" of providing safe, quality, patient care and effective pain management, these "patients" flow through all the care delivery "processes" that occur within the inpatient setting. Varying "providers or professionals" provide care based upon processes developed within the organization. Through the course of stay, "patterns" emerge regarding the effectiveness of that care. The microsystem analysis provided detailed information that was used to develop and refine the MFI and PDSA process that followed.

The MFI and PDSA process specific to this case study involved the following steps. A task force was chartered and the group answered the MFI three guiding questions, as well as developed the "Plan" for the improvement project, defining a specific aim. The "Do" phase of the improvement project involved several critical steps, which included the microsystem analysis which provided quantifiable data for the project, a literature appraisal, a policy analysis, flow charting, and an indirect event analysis. In the "Study" phase of the project, descriptive statistics were reviewed and studied for patterns. These data were collected during multiple phases of the microsystem analysis and during the indirect event analysis. The final phase of the "Act" stage was to disseminate the information obtained specifically for the pain pump transfer process, as well as to develop and disseminate the process for analyzing and improving an identified system-based improvement project. The following details further describe the improvement processes used to examine the pain pump during patient transport process.

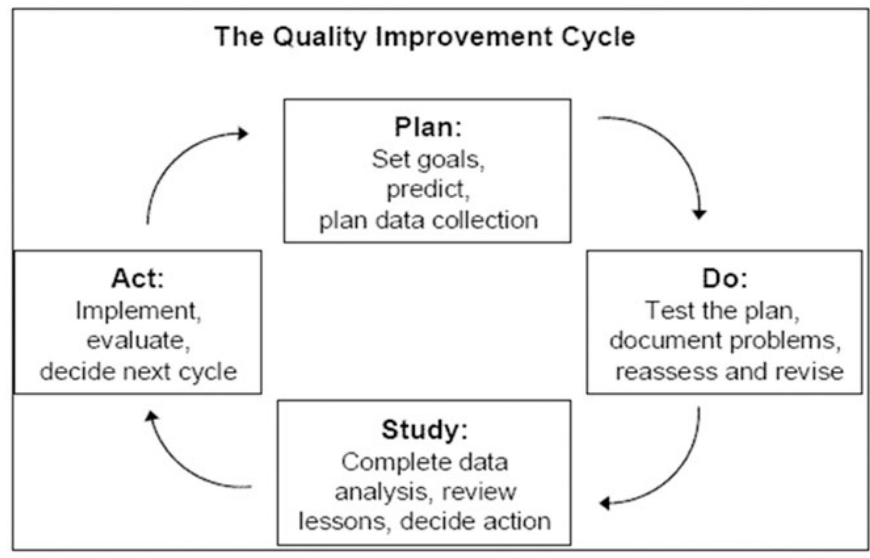

Figure 2. PDSA cycle

\subsection{Developing the perspective}

The use of PCA as an effective strategy for controlling pain within the institution under study had not been evaluated from the perspective of the processes surrounding patient transfer with a PCA pump. Due to verbal reports of variation across units in the practice of transferring patients prescribed 
a pain pump, the effectiveness of the therapy and patient outcomes may vary with inconsistencies in the processes at play in the clinical setting, thus requiring more in-depth investigation of the process. In the exemplar case, the patient was transferred without the pump that was prescribed. An assessment of the processes observed in the transfer of patients with a pain pump was the first step in the improvement process.

\subsection{Setting}

The setting for this case study is an urban academic medical center located within a university campus in the southeastern United States. The medical center is a Level 1 trauma center with over 1,100 beds offering inpatient and ambulatory services (spanning 11 city blocks). The department of nursing services exists within the bureaucratic, hierarchical structure of the academic medical center. The health care organization has 3000 nurses who contribute to the medical, surgical, psychiatry, cardiovascular, neuroscience, women's, ambulatory, and emergency services.

The academic partner site is a School of Nursing that prepares nurses at the bachelor's, master's, and doctoral levels. The program contains the state's only Doctor of Philosophy (PhD) program and a joint Doctor of Nursing Practice (DNP) program (with 2 other schools within the state system).

\section{Patients}

Building a Team to Manage A Panel of Primary Care Patients Mlssion: The Dartmouth.Hitcheock Clinic exists to serve the health care needs of our patients.

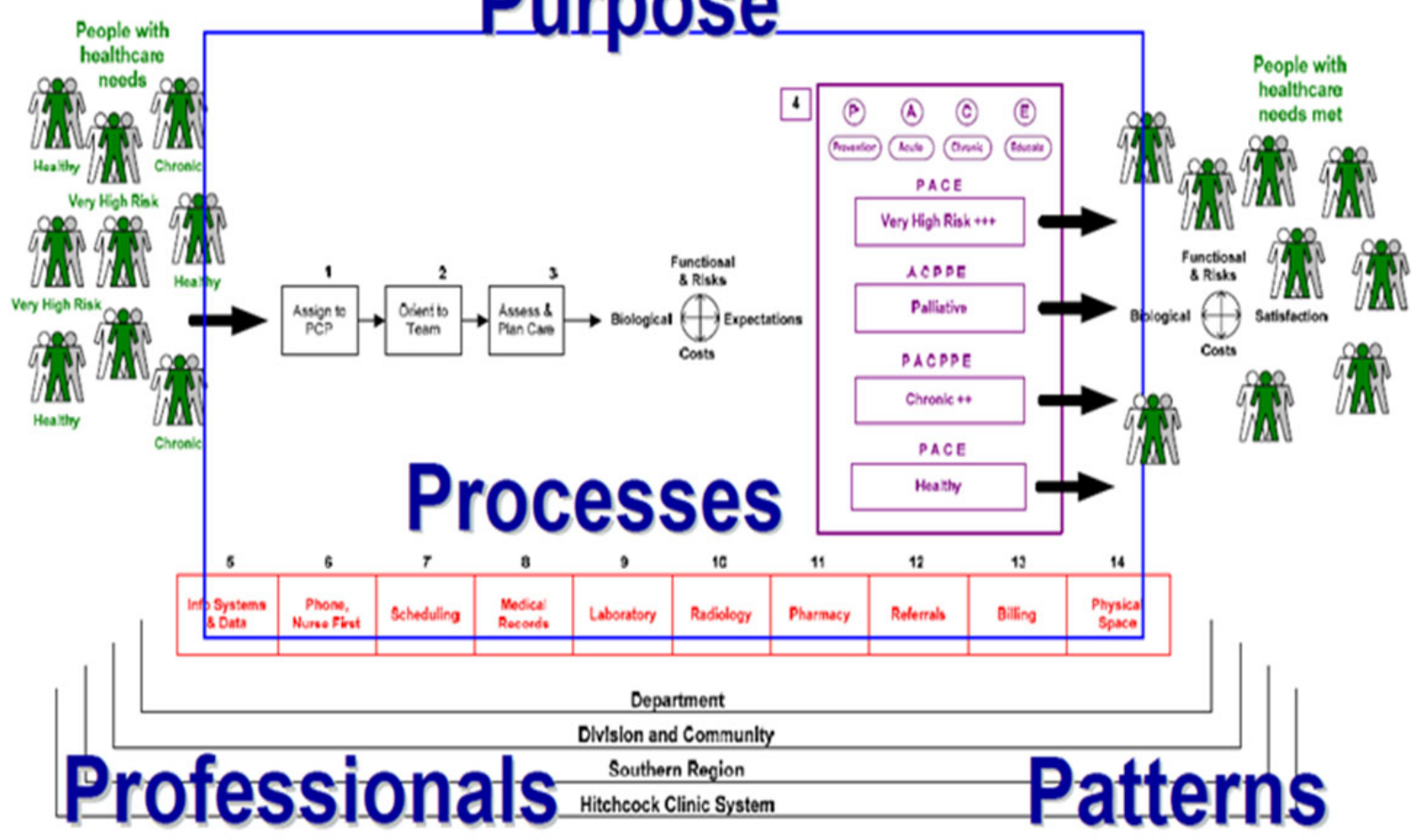

Figure 3. Microsystem analysis

2.3 Improvement and safety science: Methods for understanding the whys and decreasing variation

A task force was convened to examine the transfer process for patients with a pain pump. The organization of the group was defined through methods steeped in improvement and safety science. A nurse manager of a unit with a high utilization of pain pumps was appointed by the CNO to lead the improvement process; the nurse also happened to be a DNP student at the partner School of Nursing. The DNP program curriculum is focused on systems change, and improvement and translation sciences. The facilitator for the project was the nurse improvement scientist, whose role as a member of the CNO's staff is to provide leadership in improvement, translation, safety science, and systems change.

A formal charter was developed to guide the group through a thorough analysis of the process under review. The components of the charter included: definition of the process goals using the three guiding questions of the Model for Improvement, the microsystem analysis process and data collection specific to the process under study, an evidence table for identifying best practices in the literature specific to the pro- 
cess(es) under study, and a small test of change grid which was used to designate the following components : 1) the project plan (Plan) and timeline; 2) the components that were completed (Do); 3) the analysis of the data collected (Study); 4) recommendations (Act) for improvement, dissemination, or revision; and 5) a summation including "change theory" focused recommendations.

In addition to improvement science methods, safety science was incorporated into the charter in the form of an indirect event analysis data collection plan. Understanding the "whys" of a system variation was an important aspect to the identification of gaps in the process that resulted in systems failures or process variation. A data collection tool was developed to investigate "why" patients were not being transferred with a PCA pump, both to quantify the degree of the problem, but also to understand the systems or infrastructure surrounding the process, and where failure points may be occurring.

\subsection{Definitions}

The following definitions are integral to understanding the activities associated with the work of the task force in this case study:

Model for Improvement (MFI). The model for improvement is defined in the case study as a systematic methodological framework for improving processes and systems. ${ }^{[10]}$ Three guiding questions are used to frame the improvement project: 1) What are we trying to accomplish? 2) How will we know if a change has resulted in an improvement? and 3) What changes can we implement that will result in an improvement? The answers to these questions are then iteratively studied using the small test of change process or PDSA.

PDSA. The PDSA ${ }^{[10]}$ is defined in this case study as a small test of change cycle. The process was used to plan the work of the task force, implement the work, study the work accomplished, analyze the data collected, and then to define the next steps for systems level improvement.

Microsystem analysis. The microsystem analysis is defined in this case study as the data driven approach to analyzing the 5 P's ( purpose, patients/populations, providers, patterns, and processes) associated with the task force goals. The Dartmouth model ${ }^{[7,11]}$ for analyzing the clinical microsystem is the foundation for this activity.

Evidence table. The evidence table is defined as a structured approach to evidenced based practice that results in the review of literature surrounding a topic of interest, appraisal of the literature, and analysis of the literature to support implementing literature-supported best practices. A table is developed and used to guide improvement activities. An example of an evidence table is shown in Figure 4.

Indirect event analysis. Indirect event analysis is defined in this case study as the process for examining the "whys" of a process failure or variation. A tool is developed in order to collect information on variables specific to the process under study as well as any gaps in the process. In this case study, an excel tool was developed to collect data elements on each patient with a pain pump who were transferred for any reason during the hospital stay. Elements were collected on the following variables: patient identifier, sending unit, receiving unit, reason for transfer, "Yes/No" transfer with pain pump, and reason for "No" responses for "not transferring with a pain pump". Additional elements were collected on the entire transfer process including PCA handover/handoff information and timing elements specific to the process.

\subsection{Case study analysis: Task force aims}

The aim or goal of the task force was collectively determined to be the delivery of safe, timely, effective, efficient, equitable, patient centered care as defined by the Institute of Medicine (IOM) goals for Quality and thus amenable to the pain management process and specific to PCA use. ${ }^{[12]}$ In order to achieve this goal, it was determined that patientcentered care would be most optimally achieved if all patients in the medical center were transferred with the PCA pump. Two exclusions to transfer with a pain pump were identified: 1) a responsible, licensed provider deems it necessary to transfer a patient without a pain pump, and if this decision is necessary, the provider would discontinue the active PCA order prior to patient transfer; 2) the patient is transferred to the immediate and continuous care of an anesthesia provider who has the appropriate means to provide adequate pain control in the absence of a PCA pump.

\subsection{Case study analysis: Literature review and ap- praisal}

A critical appraisal of the available literature review was completed to ensure that the analysis would be steeped in evidenced-based practice as an underlying foundation for the analysis, as well as any intervention that may be developed. An evidence table was developed by the organization and used to appraise the current literature associated with the "pain pump" and "patient transport" process within an inpatient environment. The evidence table (see Figure 4) provided structure for the literature reviewed and contained the following table columns: citation, design/methods, sample/setting, major variables studied, measurement, data analysis, findings, level of evidence, and appraisal of worth to practice. 


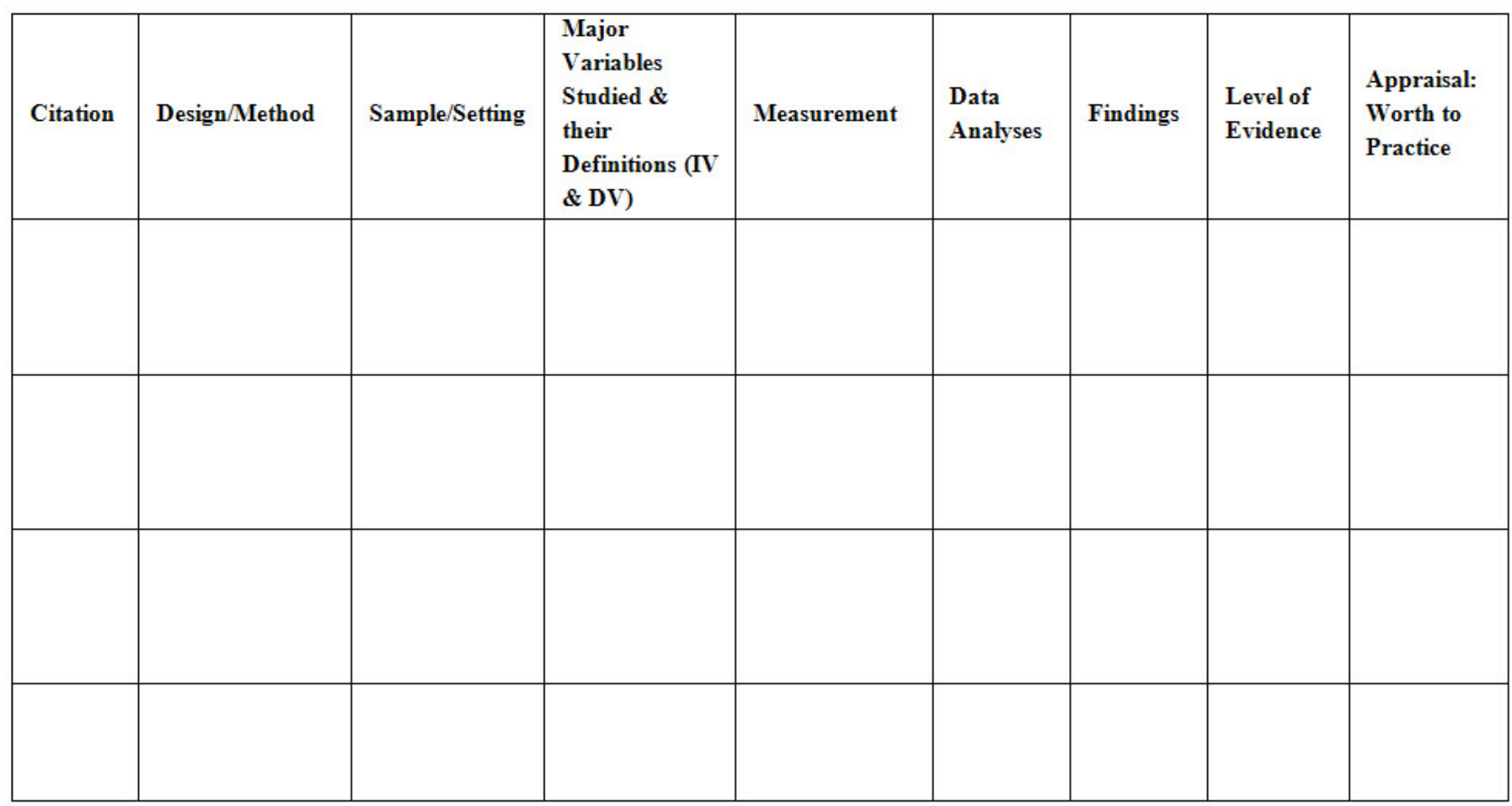

Figure 4. Evidence table

A search of PubMed and Cumulative Index for Nursing and Allied Health Literature (CINAHL) ${ }^{[13,14]}$ was completed using the search terms "pain pumps" and "patient transfer", as well as generic searches on "pain pumps" and "patient controlled analgesia". Refinements of the searches limited the results to studies published within the last five years and which were focused on human subjects.

Review of the search results led to a final analysis of over 300 articles, only six of which had information specific to patient care processes. Of these six articles, none provided a specific "best practice" method for transfer of a patient with a pain pump. In addition, higher level evidence that was "nursing process specific" was not found. Three of the articles were descriptive studies and one provided content for continuing education in nursing.

However, it is clear from the review that patient assessment and processes supporting safe patient care of patients using patient controlled analgesia is a central theme for the management of the patients utilizing this type of pharmacological therapy.

\subsection{Case study analysis: Patterns and processes}

The task force examined patterns and processes of care associated with the transfer process of a patient with a pain pump. Patterns examined were specific to data associated with the process. It was assumed by the task force that the following data patterns were directly or indirectly associated with the process: 1) patient satisfaction scores within the
HCAHPS pain management domain as a measure of the effectiveness of continuous pain control through the transfer process; 2) volume-based data on the highest utilization of pain pump as evidenced through units with the highest PCA orders as the initial focus for standardization and process development; 3) data patterns associated with safety of the PCA process, specifically prescriber medication reconciliation rates on patient transfer; and 4) data associated with the transfer process specific to the reasons for transfer and the "whys" of failing to transfer a patient without a pain pump.

Table 1 depicts the highest volume areas for "PCA orders" in a given week. The table was used to ensure that representation from the highest volume units was available to confirm and validate the patterns identified, as well as to assist with any improvement efforts.

Processes were analyzed by the task force using process mapping in order to provide a visual view of the standards and policies associated with the transfer of patients with a pain pump. Specifically, standards included in the process mapping were: 1) transfer of the inpatient (sections specific to orders and pain pumps); 2) controlled drug policies; 3) medication reconciliation; 4) handoffs; and 5) Lippincott standards ${ }^{[5]}$ associated with nursing care and the management of pain. The task force used process mapping in order to understand the broader context of pain management, and to understand where the transfer of the patient with a pain pump fit within the broader context. In addition, this broader view was used to caution the task force about inclusion of 
other working groups in the process, as changes in one area of a larger process will ultimately impact other processes.

Table 1. Highest volume PCA orders by unit per week

\begin{tabular}{|c|c|c|}
\hline \multirow{2}{*}{ Unit } & \multicolumn{2}{|c|}{ PCA Orders } \\
\hline & $\mathbf{N}$ & $\%$ \\
\hline GYNX & 1 & .21 \\
\hline S9SW & 1 & .21 \\
\hline 5North & 2 & .41 \\
\hline Emergency Department & 2 & .41 \\
\hline HVC & 2 & .41 \\
\hline WPAC & 2 & .41 \\
\hline HTIC & 3 & .62 \\
\hline P8 & 3 & .62 \\
\hline P9 & 3 & .62 \\
\hline UNSN & 3 & .62 \\
\hline 5 Main & 4 & .82 \\
\hline ICU & 4 & .82 \\
\hline P7 & 5 & 1.0 \\
\hline Blank/Unknown & 6 & 1.2 \\
\hline BMT & 6 & 1.2 \\
\hline LDR & 6 & 1.2 \\
\hline $\mathrm{MBU}$ & 6 & 1.2 \\
\hline S8S & 7 & 1.4 \\
\hline S9SE & 7 & 1.4 \\
\hline SUR5 & 7 & 1.4 \\
\hline $\mathrm{CCU}$ & 8 & 1.6 \\
\hline M7 & 8 & 1.6 \\
\hline PAC5 & 9 & 1.9 \\
\hline Prep Hold 5 & 9 & 1.9 \\
\hline HOSU & 10 & 2.0 \\
\hline PCCU & 10 & 2.0 \\
\hline Prep Hold 7 & 10 & 2.0 \\
\hline NICU & 11 & 2.3 \\
\hline OSCU & 11 & 2.3 \\
\hline TBNU & 11 & 2.3 \\
\hline University ED & 11 & 2.3 \\
\hline ATCU & 14 & 2.9 \\
\hline Post Anesthesia Holding & 14 & 2.9 \\
\hline CICU & 17 & 3.5 \\
\hline MICU & 21 & 4.3 \\
\hline W7N & 26 & 5.4 \\
\hline PAC7 & 29 & 6.0 \\
\hline Same Day Surgery & 29 & 6.0 \\
\hline SUR7 & 38 & 7.8 \\
\hline SICU & 42 & 8.7 \\
\hline TBIC & 67 & 13.8 \\
\hline Total & 485 & \\
\hline
\end{tabular}

Published by Sciedu Press
Through the pattern and process analysis, it was validated that there was variation or gaps in the process due in part to variations in standards or processes. It was discovered that the language and processes defined in the standards or procedures were not consistent across, and in certain cases were contradictory, to other standards or policies (see Table 2 ). In addition, the method for updating and communicating standards or policy changes was inconsistent, therefore contributing to variation in staff understanding of the most up to date processes.

The number of patient transfers with and without pain pumps are summarized in Table 3. Only 4 (7.7\%) of the 53 patients were transferred without their pain pumps. Data patterns around the "whys" of failing to transfer a patient with a pain pump were not large in volume due to a small sample size (n =53), however, this was due in part to the fact that the data collection period was limited to one week for rapid cycle improvement. However, the data collected provided a level of understanding of factors contributing to the failures in the process of transferring patients with a pain pump.

For those cases where patients were transferred with pain pump, the reasons for doing so are summarized in Table 4. As noted below, the reasons are varied and no consistent pattern is evident. This is not surprising given the small number of transfers without pumps. However, miscommunication, lack of equipment, errors in omission in setting up the order for a pain pump, and provider decisions about ease of transport were factors identified as impacting the transfer process of patients with a pain pump.

\subsection{Case study analysis: Summation and recommenda- tions}

The task force concluded the chartered work within 5 weeks, with one week being designated for data collection on the units using the indirect event analysis tool. The recommendations of the task force focused on aligning hospital standards and policies, effective communication of said policies/standards, and leadership accountability associated with patient centered care and adherence to the policies/standards for the transfer of patients with a pain pump. Specific language was crafted and submitted to the regulatory group within the institution for the alignment and integration within organizational policies, procedures, and standards. In addition, during the analysis and examination of the processes, several incidental findings were identified. One such finding was a gap in the data collection process for medication reconciliation at transfer. The development of an effective reporting tool will need to be developed to determine the status of the prescriber reconciliation process as the nursing process is focused on "counting" of controlled medications and not assessing the "need" for the therapy or medication. 
Table 2. Gap analysis-policy/standards analysis

\begin{tabular}{|c|c|c|c|c|}
\hline & $\begin{array}{l}\text { Transfer of Inpatient } \\
\text { Not cross referenced with } \\
\text { other policies/standards }\end{array}$ & $\begin{array}{l}\text { Controlled Drug } \\
\text { Not cross referenced with } \\
\text { other policies/standards }\end{array}$ & $\begin{array}{l}\text { Nursing Care and } \\
\text { Management of Patient in Pain } \\
\text { Not cross referenced with other } \\
\text { policies/standards }\end{array}$ & $\begin{array}{l}\text { Medication } \\
\text { Reconciliation } \\
\text { Not cross referenced with } \\
\text { other policies/standards }\end{array}$ \\
\hline $\begin{array}{l}\text { Stated } \\
\text { Transfer with } \\
\text { Pain Pump }\end{array}$ & \begin{tabular}{ll}
\multicolumn{2}{l}{ Variation: } \\
1) Do Not Interrupt pain \\
pump during transfer \\
2) Discontinue orders prior \\
to transfer
\end{tabular} & $\begin{array}{l}\text { Variation: } \\
\text { Do Not Discontinue } \\
\text { orders prior to } \\
\text { transfer }\end{array}$ & Does Not Address & Does Not Address \\
\hline $\begin{array}{l}\text { Narcotic } \\
\text { Reconciliation }\end{array}$ & $\begin{array}{l}\text { Nursing counts with paper } \\
\text { documentation } \\
\text { (outdated, did not update with } \\
\text { implementation of EMR) }\end{array}$ & $\begin{array}{l}\text { Nursing count with } \\
\text { paper/EMR documentation } \\
\text { (newly revised standard) }\end{array}$ & Global language & Does Not Address \\
\hline $\begin{array}{l}\text { Medication } \\
\text { Reconciliation }\end{array}$ & Does Not Address & Does Not Address & Does Not address & $\begin{array}{l}\text { Reconciliation at } \\
\text { admission, transfer, } \\
\text { discharge }\end{array}$ \\
\hline
\end{tabular}

Table 3. Frequency of transfers with and without pain pumps

\begin{tabular}{lll}
\hline Pain Pump on Transfer & \multicolumn{2}{l}{} \\
\cline { 2 - 3 } Yes/No & $\mathbf{N}$ & $\mathbf{\%}$ \\
\hline Yes & 4 & 7.7 \\
No & 48 & 92.3 \\
Total & $* 52$ & \\
\hline
\end{tabular}

*Reviewed 53 transfers, 1 record missing data for the element "Pain Pump on Transfer”.

Table 4. Reasons for "No" transfer with pump (Failure to transport with pain pump

\begin{tabular}{lll}
\hline Reason for “No" Pain Pump on & \multicolumn{2}{l}{ Frequency/Percentage } \\
\cline { 2 - 3 } Transfer & $\mathbf{N}$ & $\mathbf{\%}$ \\
\hline Ease of Transport/Short Trip & 1 & 1.9 \\
Miscommunication & 1 & 1.9 \\
No Pumps Available & 1 & 1.9 \\
PACU Did Not Set Up & 1 & 1.9 \\
Total Transports & $\mathbf{5 3}$ & \\
\hline
\end{tabular}

\section{Discussion}

The results of the analysis of the pain pump transfer project will be discussed from the perspective of the following: system-based improvement, leadership accountability, and education-practice partnerships.

This systems-based improvement project was organized based upon information obtained by the $\mathrm{CNO}$ regarding what appeared to be a simple process variation. However, upon chartering a clinical team to systematically examine the pain pump transfer process it was determined that there were systems opportunities somewhat unrelated to individual variation by a provider. Such variation was a key point for impacting organizational learning. Often there is a rush to "fix" a problem before an examination of the underlying opportunities that may exist.

The literature appraisal of "pain pump" and "patient transport" did not reveal significant support in the literature for a standardized approach to managing the "transfer" process with a pain pump. The literature did support safe pain care for patients using this form of pain management, through effective nursing care delivery and assessment. However, the organizational approach to the process was not necessarily a gap. The gap in the process was created by a failure to standardize the process and provide clear communication to the nursing staff on the most current process as defined within the organizational policies, procedures, and standards.

In this situation there were structural opportunities regarding leadership accountability with process variation. There were also opportunities surrounding the processes for implementing and communicating standards and policies when changes occur. Both of these outcomes warrant broader leadership engagement. Revising policies and standards is a major endeavor that requires the coordination of multiple groups and individuals in order to meet organizational needs while also ensuring that regulatory requirements are met. Communication of policies and standards is challenging, as there is no single method that has been found to be effective in communication of critical information to all individuals at all levels of the organization.

This project also highlighted the need to examine systems opportunities using a structured method with a designated facilitator and defined tools. The use of the "indirect event analysis" process described is a method that is particularly effective for examining the "whys" of process failures that are occurring frequently at the unit level, but would perhaps not "bubble" up to a broader scale organizational event anal- 
ysis (or root cause analysis). In this case study, information received by the CNO suggested that the failure to transport a patient with a pain pump was a frequent occurrence. However, the data collected during the week long data collection period indicated that the rate was relatively low with a $7.7 \%$ failure rate. Thus the actual number of failures in transferring patients with a pump was not nearly as large as was initially expected. However, any rate of failure may contribute to less desirable patient outcomes, as well as poorer patient satisfaction with care.

Finally, this project highlights the importance and the success of an education and practice partnership. The DNP is a practice doctorate, focused on systems improvement. In this institution there is a desire to more fully engage students in the academic setting with projects that exemplify utility in the practice realm. Nursing system leadership and nurse educational administration have sought a format that will build this type of relationship. Such relationships not only create a positive learning environment for students, but also support an organization where nursing leads change as a single entity. Combining the school of nursing faculty expertise in facilitating the improvement process with the nursing staff's expertise of the clinical environment, creates a "win-win" partnership for facilitating systems change.

\section{Conclusions}

Managing the transport of patients with a pain pump within a healthcare organization is a process that should be standardized and effectively communicated to ensure the understanding of all who need to know because of their role in providing care to patients. The standardization of care based on best practices contributes to safer care and greater patient satisfaction. However, many factors can contribute to gaps in knowledge and understanding of standards that can result in poor outcomes. This case study and system-based improvement project highlighted the need within an organization to develop a standardized process for learning from process variation, and also for developing the tools and competencies within the organization for this work. In particular, the nursing workforce can play a primary role in leading and managing continuous improvement. Nurses are at the front line of care, and as such, are optimally positioned to identify improvement opportunities, as well as to provide the ideas for improving process and system. To assume this role, nurses must be engaged in the process of developing improvement and safety competencies within the nursing workforce in addition to the basic QSEN competencies ${ }^{[15]}$ that are embedded within nursing education. In addition, the development of such competencies support the work of a Magnet ${ }^{[16]}$ designated organization. A standardized process, such as the one used in this case study, may be replicated for any improvement identified in the delivery of care, and can be used by any inter-professional team given the proper leadership. Understanding the underlying process or gap begins with analysis before action, and intervention begins with evidence before action.

\section{ACKNOWLEDGeMENTS}

The authors would like to acknowledge Karen Harris, MSN, RN, OCN, CCM, Nurse Manager and DNP student within the study organization for her leadership in bringing together the inter-professional clinical team.

\section{CONFLicts OF INTEREST Disclosure}

The authors declare that there is no conflict of interest statement.

\section{REFERENCES}

[1] Melzack R. The Tragedy of Needless Pain. Scientific American. 1990; 262(2): 27-33. PMid:2296714 http://dx.doi.org/10.1038/s cientificamerican0290-27

[2] Chumbley G, Mountfourd L. Patient-controlled analgesia infusion pumps for adults. Nursing Standard. 2010; 25(8): 35-40. http: //dx.doi.org/10.7748/ns2010.10.25.8.35.c8067

[3] Chumbley G. Patient-Controlled Analgesia. In Perioperative Pain Management. Oxford: Blackwell Publishing; 2009.

[4] Hudcova J, McNicol E, Quah C, et al. Patient Controlled Opioid Analgesia versus conventional opioid analgesia for postoperative pain. Cochrane Database for Systematic Reviews. 2006. PMid:17054167 http://dx.doi.org/10.1002/14651858.CD003348.pub2

[5] Nettina S. Lippincott Manual of Nursing Practice (10th ed.). Philadelphia, Pennsylvania: Wolters Kluwer Health/Lippincott Williams \& Wilkins; 2014

Published by Sciedu Press
[6] CMS: HCAHS: patients' perspectives of care survey [Internet] Baltimore: Centers for Medicare and Medicaid Services; [cited 2015]. Available from: http://www.cms.gov/Medicare/Quali ty-Initiatives-Patient-Assessment-Instruments/Hos pitalQualityInits/HospitalHCAHPS.html

[7] Nelson E, Batalden P, Godfry M, et al. Value by Design: Developing Clinical Microsystems to Achieve Organizational Excellence. San Francisco: Jossey-Bass. 2011.

[8] Langley G, Moen R, Nolan K, et al. The Improvement Guide: A Practical Approach to Enhancing Organizational Performance (2nd ed.). San Francisco: Jossey-Bass; 2009.

[9] Sherwood G, Barnsteiner J. Quality and Safety in Nursing: A Competency Approach to Improving Outcomes. Oxford: Wiley-Blackwell; 2012.

[10] API: associates in process improvement. [cited 2015 April 6]. Available from: http://www.apiweb.org/ 
[11] Microsystem Academy: transforming microsystems in healthcare. [cited 2015 April 6]. Available from: https ://clinicalmicros ystem.org/

[12] IOM: crossing the quality chasm: the IOM healthcare quality initiative. [cited 2015 April 6]. Available from: http://www.iom. edu/Global/NewsAnnouncements/Crossing-the-Quality -Chasm-The-IOM-Health-Care-Quality-Initiative.aspx

[13] Bethesda (MD): National Library of Medicine (US). 1997 June. Available from: http://www.ncbi.nlm.nih.gov/pubmed
[14] CINAHL Database [Internet]. EBSCO (US). [Cited 2015]. Available from: http://www.ebscohost.com/nursing/products /cinahl-databases/cinahl-complete

[15] QSEN: quality and safety education in nursing: [cited 2015 April 6]. Available from: http://qsen.org/

[16] ANCC: American nurses credentialing center. [cited 2015 April 6]. Available from: http://www. nursecredentialing.org/magne t. aspx 